\title{
APORTES DESDE LOS MÁRGENES A LA CO-CREACIÓN DE PRÁCTICAS OTRAS DE CONOCIMIENTOS
}

\section{Contributions from the Margins to Co-creation of Other Knowledge Practices}

\section{XOCHITL LEYVA SOLANO*}

Fecha de recepción: 13 de junio de 2021 - Fecha de aprobación: 30 de agosto de 2021

\section{Resumen:}

Mi punto de partida son los despojos epistémicos que trajo consigo el disciplinamiento antropológico. Me enfoco en las prácticas otras de conocimientos y las contribuciones que hacen a las ciencias sociales las mujeres y otres racializadas rebeldes en los actuales tiempos de violencia y muerte. De la mano de comunicadoras comunitarias indígenas y garífunas abordo las formas otras de entender lo que en términos académicos llamamos "comunicación". Reflexiono sobre algunos aportes epistémico-teórico-políticos zapatistas efectuados en medio de las guerras. Cierro sugiriendo que todas estas luchas epistémico-políticas pueden verse como un aporte colectivo desde los márgenes a eso que aún llamamos antropologías latinoamericanas.

Palabras clave: despojos epistémicos, disciplinamiento, sujetos(as) epistémicas, prácticas otras de conocimientos, luchas epistémico-políticas.

\section{Abstract:}

My starting point is the epistemic dispossession that anthropological thought and discipline has brought with it. I will focus on other knowledge practices and the contributions that women, trans-gender and racialized others have made to the social sciences in our current times that are marked by widespread violence and death. Based on our work with indigenous and Garifuna community communicators, I address other ways of understanding what, in academic terms, we call "communication". This leads me to reflect on some of the Zapatista epistemic-theoretical-political contributions made in the midst of warfare. I conclude suggesting that all these epistemic and political struggles can be understood as a collective contribution made from the margins to what we still call Latin American anthropologies.

Keywords: epistemic dispossession, disciplinary power, epistemic subjects, other knowledge practices, political and epistemic struggles.

\footnotetext{
* Dra. en Antropología. Trabajadora de las ciencias sociales, activista de las redes altermundistas. Profesorainvestigadora, Centro de Investigaciones y Estudios Superiores en Antropología Social, sede Sureste, Chiapas, México. Correo-e: xls1994@gmail.com
} 


\section{Introducción}

Cuando vi el título del dossier dudé sobre si yo era la persona más indicada para escribir sobre las contribuciones de la "antropología latinoamericana". Si bien es cierto que fui formada en la disciplina antropológica, desde el alzamiento zapatista de enero de 1994 he venido, poco a poco, y de la mano de las mujeres, Ixs ${ }^{1}$ jóvenxs, les otres y los pueblos en resistencia, cuestionando la herencia colonial y (hetero)patriarcal de lo que fue el sustento de mi disciplinamiento. A estas alturas no me defino como "antropóloga", prefiero nombrarme como "trabajadora de las ciencias sociales, activista de las redes altermundistas". No se trata de un simple giro semántico sino más bien onto-epistémico-político-teórico-práctico que me ha costado mucho dolor, angustia, ruptura, rabia, pero también me ha llevado a momentos de exploración, descubrimiento y luminosas convergencias. Todo ello ha pasado en los últimos cuarenta y dos años.

Uno de esos descubrimientos dolorosos tuvo que ver con el sentido colonial y (hetero) patriarcal del término América Latina. Eso fue duro. América Latina para mí era una categoría política potente que evocaba todo lo que nos pasó en las décadas de 1970 y 1980: el sentido de solidaridad con los refugiados llegados a México desde Sudamérica, el rechazo a las dictaduras del sur, el efecto de la Revolución cubana y de las luchas de liberación en Centroamérica. Ese era, de una u otra forma, mi mundo hasta que empecé a leer la crítica decolonial al término Américo/a Latin/a y luego empecé a saber más y más de las impugnaciones que este tenía desde Améfrica ladina y desde lo que hoy se nombra como Abya Yala. Con todo esto no quiero decir que debemos tirar a la basura este término, sino que hay que relativizarlo, mirar sus alcances y sus límites. Y, sobre todo, desde quién, por qué y para qué es cuestionado y puesto en tela de juicio.

Dicho lo anterior tendría entonces que refrasear la pregunta que nos lanzaron los editores de este dossier y más bien centrarme en compartirles cómo la antropología como disciplina pasó, en mi/nuestra vida, a ser una herramienta, un instrumento, un arma de lucha por la vida digna, justa y plena para todxs. De eso se trata este texto, de recopilar fragmentos del cómo, dónde, por qué, cuándo, con quién y contra quién fui/mos encarnando colectivamente este proceso de corte despatriarcalizador y descolonizador. $Y$ si bien es cierto que esto pasó en lo que tradicionalmente concebimos como América Latina y el Caribe, hoy, en 2021, nos movemos en un terreno glocal que trasciende las fronteras convencionales de los Estados-nación y más bien operamos en/desde las redes globales contrahegemónicas, también llamadas de la esperanza.

En la primera sección de este texto hablaré de algunos despojos epistémicos que trajo consigo el disciplinamiento antropológico. En la segunda abordaré lo que he llamado los aportes de las prácticas otras de conocimientos (así en plural). En la tercera visibilizaré las contribuciones que hacen, en tiempos de muerte, a las ciencias sociales las mujeres y otres racializadas rebeldes. En la cuarta parte, de la mano de comunicadoras comunitarias indígenas y garífunas, abordaré las formas otras de entender lo que en académico llamamos "comunicación". En la quinta parte navegaré con las, los y loas zapatistas para dejar constancia de algunos de sus aportes epistémico-teórico-políticos en medio de las guerras (así en plural). 
Cierro sugiriendo que todo este caminar podría verse como un aporte colectivo desde abajo y a la izquierda, desde los márgenes, a eso que conocemos como antropologías latinoamericanas (así en plural). Corto fragmentos de un largo viaje de más de cuatro décadas donde hemos buscado desdisciplinar la antropología a cien años de que entró al santuario de las disciplinas sociales. Lo hicimos empujadas por las resistencias alter $y$ anti y por el sincero interés de volverla útil a los sujetos y sujetas con los que comulgamos hoy en esta ardua, cruenta y dolorosa lucha por la vida. Este viaje lo hemos emprendido con aquellos(as) a quienes el lenguaje colonial imperial ha reducido a "objetos de estudio", reducido al "Otro" (así en masculino y singular).

\section{Los despojos epistémicos del disciplinamiento ${ }^{2}$}

Las disciplinas de las ciencias sociales que aprendemos en las universidades se llaman así no por casualidad. A través de ellas nos re-educan y se nos ponen unos lentes para mirar y actuar en eso que llamamos "realidad". Nuestra entrada a la educación escolarizada $y$, por ende, a la universidad, es un proceso complejo que se vive, en cada caso, de manera diferente, pero comparte elementos sistémicos, estructurales, culturales y coyunturales. Por el momento quiero mirar algunas de las aristas de ese complejo devenir, aunque reconozco que no alcanzaré, en esta pequeña sección, a abordar todos los colores de la complejidad de ese fenómeno. Me centraré en algunos aspectos para sustentar mi argumento. Una disculpa de antemano si lo que digo suena demasiado parcial, pero necesito visibilizarlo para que tenga un efecto catalizador.
En un primer momento quiero destacar los despojos no de los Otros sino de nosotrxs Ixs universitarixs. Despojos a los que somos sometidas al vernos encajonadas en una corriente, una teoría o un "marco teórico". Para evitar que ese despojo se consuma no voy en este texto a apelar ni a corrientes ni a teorías, por lo menos no en la forma convencional. No lo haré porque pienso que sería reproducir los despojos heterónomos normalizados y legitimados por el sistema académico que operan como si hubiera una voz en off que te dice qué pensar, cómo expresarlo y qué expresar. Despojos hasta cierto punto epistemicidas donde lo que se mata es la posibilidad creativa autónoma de expresar de manera clara y sencilla lo que nos es urgente o, mejor dicho, "las palabras" y "las cosas" que emergen del diálogo con la gente con la que trabajamos, vivimos y comulgamos: en mi/ nuestro caso, mujeres y jóvenes(as) campesinas mayas del Sur profundo.

Desde hace rato dejé/dejamos de escribir únicamente para una élite universitaria letrada. Nos negamos a escribir en ese formato críptico lleno de autores, teorías y citaciones que solo le interesan a y pueden ser entendidas por un puñado de iniciados. Nuestra rebeldía se ratifica cuando vemos libros de famosos investigadores tirados, enlodados, deshojados en el traspatio de la casa de los campesinos con quienes vivimos. Paradas frente a lo que a nosotras nos parecía un sacrilegio, preguntamos al campesino que nos hospedaba: "¿Y ese libro?" Obtuvimos una sencilla respuesta: "fue un regalo". Nada más, no hubo ningún intento de rescate del mismo. En ese contexto nos quedó más que claro lo que ya sabíamos: el valor del libro académico en contextos rurales, campesinos o populares. A los libros de texto no les va mejor, acaban usados como papel de baño en la letrina. 
Dicho lo anterior, viene a nuestra mente una pregunta básica: ¿cuándo fuimos despojados de la posibilidad de encontrarnos de verdad entre ellxs y nosotrxs? Pensemos en el juego perverso de nuestra sociedad "moderna" que separa el trabajo intelectual del manual, tocándonos a nosotras el primer cajón y a ellos el segundo. Los "pueblos sin historia" o reducidos a historia oral, mientras nosotras gozamos del aura teórica de los letrados. Así pasan décadas y más décadas y seguimos viendo llegar a los territorios mayas a muchos universitarios solidarios con sus teorías y autores académicos a cuestas y quedarse mudos ante nuestra pregunta: ¿además de leer y escribir, qué cosas prácticas sabes hacer? Silencio...

La educación escolarizada ha despojado a una parte importante de la humanidad de sus creatividades autónomas, de hecho, requeriríamos la misma cantidad de siglos para reparar ese despojo y aún así, tal vez, no lo lograríamos, porque se ha aceptado como moneda corriente ejercer la violencia de la interpretación ${ }^{3}$ y se ha naturalizado la violencia epistémica; ingredientes sine qua non del capitalismo, el heteropatriarcado y el racismo. De hecho, ya solo usando toda esa terminología ejercemos un buen tanto de esas violencias a las que podríamos llamar -en nuestra jerga críptica- "epistémicas". Parecería imposible salir de los círculos de esas violencias generadas por nuestra socialización/domesticación/disciplinamiento para pensar, interpretar, analizar y actuar.

En las secciones siguientes abordaré cómo, en lo personal y colectivo, he/mos buscado salidas a esa espiral de violencias y despojos. Esto no quiere decir que lo he/mos logrado, pero sí lo he/mos intentado y lo seguimos intentando. De hecho, una vía para combatir La ciudad letrada
(Rama, 1998) ha sido la fundación, hace 21 años, del Proyecto Videoastas Indígenas de la Frontera Sur (PVIFS) para coadyuvar en la creación de medios audiovisuales propios de los pueblos en resistencia. En esos medios se habla de las cosas urgentes e importantes para la gente con quienes convivimos y colaboramos. Desde hace dos años, unos meses antes de que entrara el COVID-19 a México, mi compañero de vida y yo nos fuimos a vivir a un territorio maya en resistencia, no para "hacer trabajo de campo" o para "investigarlos", sino para caminar con ellxs la educación y la salud autónoma, así como la investigación desde y para su gobierno comunitario y de esa manera fortalecer su autonomía. Autonomía que viven de facto en muchos sentidos y que exigen de jure desde 2017 ante los tribunales correspondientes que marca la limitada ley mexicana. Desde ese vivir juntas se han dado nuevas posibilidades de construir una nosotridad entendida como seres vivos co-existiendo en medio del colapso civilizatorio pandémico, re-existiendo y luchando por la vida digna, justa y plena para todxs. Ahí vuelve a abrirse la posibilidad de seguir caminando la antropología como herramienta/medio/arma antes que como disciplina disciplinante ${ }^{4}$.

\section{Prácticas otras de conocimientos, entre crisis y entre guerras}

Algo que se naturaliza en el proceso de disciplinamiento al que somos sometidas es el pensar desde un norcentrismo-eurocentrismo puesto en un eterno masculinismo abstracto emitido desde ningún lugar para así lograr la "objetividad": la cúspide del pensamiento positivista y neopositivista tan ampliamente criticado a lo largo, sobre todo, de la segunda mitad del 
siglo $X X$ y lo que va del $X X I$. Hoy en día, sin embargo, encontramos investigaciones que no se han enterado de esa abundante y diversa crítica. En reacción a todo ese disciplinamiento e impactada por los efectos rebeldes del zapatismo, es que llegué/llegamos en 2008 a la necesidad de rastrear a detalle qué estaba pasando con esa crítica y con la insurrección de saberes subyugados en/desde el Sur profundo.

Para muchos colegas dicha insurrección se reducía a los estudios poscoloniales, para otras a las epistemologías feministas, para otros a la investigación-acción participativa (IAP), a la antropología colaborativa o a la antropología militante. Todas ellas eran aportes reales e importantes, pero parte de un menú muchísimo más amplio que no se constreñía a lo reconocido por la Academia (así con mayúscula y en singular). Por el caminar en diferentes territorios indígenas y afrodescendientes sabíamos que había más, mucho más, así que por siete años me encargué/nos encargamos, un puñado de miembros de la Red Trasnacional Otros Saberes (Retos), de localizar, visibilizar y tejer lo más que pudimos. Los resultados fueron impresionantes, no solo por lo que encontramos sino porque nos mostraba lo ignorante que éramos y la forma en que de nuevo el racismo epistémico y el epistemicidio operaban en las aulas universitarias y en nuestros centros de investigación. Racismo epistémico persistente aún cuando pregonábamos que ya sabíamos algo. Ese algo, era/es una pequeñísima parte de todo lo que existe. De esas pesquisas personales-colectivas salieron 1500 páginas que tomaron la forma de tres libros creados autónomamente con los que arrancamos nuestra Cooperativa Editorial Retos ${ }^{5}$. Al faro zapatista íbamos identificando, visibilizando y tejiendo resistencias epistémico-teórico-políticas desde los pueblos en pie de lucha y, al mismo tiempo, encarnábamos la autonomía en nuestro propio territorio: el disputado campo de las ciencias sociales.

¿Cómo nombrarle a eso que íbamos descubriendo? ¿Cómo hacerlo sin caer de nuevo en la clásica actitud propia del conocimiento disciplinado de meter todo en una cajita previamente hecha por algún norcéntrico investigador? ¿Cómo no terminar generalizando y nombrando desde arriba una diversidad de haceres sentipensados a ras de suelo gestados en medio de insurrecciones, revueltas, rebeliones, resistencias de pueblos indígenas y afrodescendientes? Bueno, lo primero fue respetar la diversidad de nombres que cada una de esas comunidades, colectivos, movimientos y redes le dio a lo que hacían. Eso era/es muy disruptor para el establishment que espera generes una nueva etiqueta que luego se consumirá en el templo del saber como propiedad intelectual de alguien. Yo iba en sentido contrario al respetar las formas diversas de nombrarse de todas esas 52 autorías colectivas y/o personales.

El asunto se complicó cuando alguien dijo: "¿pero de qué se trata esta colección de tres tomos?" Ahí vi la importancia de identificar un hilo conductor y accedí a ponerle, juntando tradiciones de nombrar campesinas, indígenas, académicas y feministas, prácticas otras de conocimientos (todo pluralizado). Lo que pasó luego no sé si fue bueno o malo: el término no fue retomado por nadie, nadie lo cita, ni aparece en primer lugar en ninguna de esas listas con las que se mide el rating de quotations. Las razones pueden ser muchas: desde lo poco creativo del término hasta quién lo enuncia, desde dónde se enuncia, la editorial que lo publica, el idioma en que está escrito o quiénes lo "consumen". Pero 
contrariamente a ese hecho, la obra colectiva ha caminado por todo el mundo en las maletas de amplias redes de solidaridad que se encuentran, como nosotras, disputando el territorio que ya les mencioné. El efecto de lo compilado en su conjunto y uno por uno de los 52 autores(as) sí que ha hecho mella en la Academia y en los espacios activistas. Aunque también debo mencionar que ha habido lectores que nunca han pasado de leer la Presentación y el Prólogo, mostrando así su racismo epistémico con las autoras y autores de las 1480 restantes páginas. Por desgracia, eso no es algo nuevo, sabemos que así opera el marketing académico y la jerarquización del saber/poder científico.

Detengámonos en el contenido de la obra colegiada. En ella, las crisis y las guerras, las insurgencias y las contrainsurgencias tomaron una dimensión inimaginable y se fueron yuxtaponiendo a las prácticas otras de conocimientos. En parte ello tenía que ver con lo que vivíamos en ese 2008. Los encabezados del periódico El País resumían el palpitar del momento: "El pánico hunde las bolsas. Wall Street y el resto de mercados cierran la semana con caídas superiores a las sufridas en las crisis de 1929 y 1987" (Pérez, 2008). Al principio el vínculo entre prácticas otras de conocimientos, crisis y guerras no era tan claro, pero conforme iban pasado esos siete años que les digo, la relación se fue haciendo más y más evidente en la medida en que las crisis -así en plural- se agudizaban y nos interpelaban de diferentes formas en nuestros espacios académicos y universitarios, a la vez que el capitalismo patriarcal salvaje y su concomitante colonialidad arremetían con más fuerza contra los sujetos(as)-actores(as) con los y las que trabajábamos o de los que éramos/somos parte orgánica.
Entonces empezamos a preguntarnos: ¿cómo estamos viviendo la crisis de estructuras y formas de conocimiento dominante en la Academia, en las disciplinas de las ciencias sociales y entre los(as) universitarios(as)? ¿Cómo estaba esto generando otras prácticas de conocimiento, otra geopolítica y otra economía del conocimiento? ¿Qué alternativas metodológicas, epistémicas, éticas y políticas estaban/están construyéndose en diferentes puntos del planeta Tierra? ¿Qué alcances, límites y contradicciones internas tienen dichas alternativas?

El sentido terminal de las crisis nos abría la posibilidad de revalorar las prácticas de conocimiento de aquellas personas que ya de por sí viven o han vivido más allá de la racionalidad moderna occidental. Me refiero a revalorar los aportes políticos, epistémicos, éticos, teóricos, de vida, de aquellos que han dado sustento a rebeliones, resistencias, patrones de movilización insurreccional y movimientos antisistémicos, antipatriarcales, antirracistas, antiimperialistas en diferentes momentos y partes del mundo.

Dichos patrones de movilización insurreccional hunden sus raíces en tiempos coloniales pero se refuerzan y avivan, sobre todo, en la década de 1990, cuando los pueblos originarios de Abya Yala empiezan a conformar articulaciones continentales y movimientos indígenas de alcance nacional. A partir de ese momento, estos se movilizan masivamente, practican la desobediencia civil e incluso, como sucedió en Chiapas (México), se levantan en armas con un modo muy propio: no abogan por la toma del poder sino, simplemente, por cambiar el mundo, empezando por el suyo. Todo ello sucedía al mismo tiempo que en América Latina se daba una reforma estructural capitalista neoliberal y del Estado, así como un recambio institucional. 
Con nuestras indagaciones nos estábamos acercando a las luchas políticas de los pueblos originarios y afrodescendientes, pero desde una arista emergente: la disputa explícita del sentido y la forma del saber/conocer dominante. Dicha dimensión, a la que llamé epistémica-política, crecía y se fortalecía conforme avanzaban comunidades, organizaciones y movimientos indígenas y afrodiaspóricos en sus acciones de autodefensa de su territorio y vida; conforme avanzaban sus autonomías sin permiso; sus video-autorrepresentaciones en rechazo a la heterorrepresentación; su comunicación comunitaria articulada en lo continental; la construcción de escuelas, currículos, universidades, unitierras, pluriversidades que buscan responder a sus necesidades. Las luchas epistémico-políticas en movimiento se daban en un contexto caracterizado por crisis y guerras; eran parte de un momento clave de reconstitución de los pueblos originarios y afrodescendientes.

Ellas contenían prácticas otras de conocimientos donde jugaban un papel central las mujeres indígenas organizadas, los maestros campesinos nombrados desde las propias comunidades, las y los jóvenes indígenas artistas urbanos, las y los comunicadores comunitarios y las y los indígenas académi$\cos$ (as) universitarios(as) que se atrevían a retar lo naturalizado. Pero también descubrimos que luchas epistémico-políticas similares estaban siendo libradas por los y las militantes, activistas y feministas de los colectivos, organizaciones, movimientos y redes antipatriarcales, antirracistas, anticapitalistas y antiimperialistas. Todos(as) ellos(as) de alguna manera forman parte de lo que se ha dado en llamar, en gramática académica, "las multiformes resistencias al nuevo orden panamericano", que desde comienzos de la década de 1990 han contribuido a frenar o retrasar procesos privatizadores con acciones callejeras masivas, insurrecciones armadas, derribamientos de presidentes (véase Ecuador, Argentina, Paraguay, Perú y Brasil) o el desmantelamiento de regímenes corruptos (véase Venezuela y Perú).

Lo que quedaba claro es que las insurrecciones de saberes subyugados que conllevan esos nuevos patrones de movilización estaban agrietando el patrón dominante de saber/poder. Se trataba de un agrietar desde afuera de la Academia. Dichas insurrecciones descentraban y retaban al sistema académico conduciéndonos a la búsqueda de nuevas metodologías, epistemologías, ontologías y teorías. Esas insurrecciones eran/son parte activa y prolija en la producción, construcción y creación de conocimientos otros: alter, anti, pluri, trans. A la vez, son nodos fundamentales para la construcción y creación encarnada de alternativas a la actual crisis civilizatoria.

\section{Aportes de mujeres y otres racializadas en tiempos de muerte}

Decir que el conocimiento dominante en la Academia es principalmente producido por masculinos, blancos, barbados, cisheterosexuales, adultos, posicionados o radicados en el Norte global es hoy una verdad de perogrullo en ciertos ámbitos feministas, activistas y académicos. Pero hay que volver a decirlo para ponerlo al centro del debate y porque nos permite recalcar que el disciplinamiento al que voluntariamente nos sometemos al entrar al campo de las ciencias sociales tiene color, raza, género, clase, etnia y sesgo generacional. 
Muchas veces tardamos décadas en darnos cuenta de todo eso $\mathrm{y}$, muchas otras, no logramos llegar a sentipensar encarnadamente las implicaciones que se derivan de ello. Les pongo un ejemplo. Cuando estudiaba antropología en la década de 1980 no supe que Stuart Hall era un negro de Kingston (Jamaica) llegado a Inglaterra en los años cincuenta. Sí me enteré de su filia marxista, particularmente gramsciana, de su trabajo sobre hegemonía, medios de comunicación y cultura popular, pero lo leí como si fuera un autor blanco parte de los estudios culturales. Claro, en esa época no había ni Wikipedia, ni internet para clickear y ver la foto con los rasgos fenotípicos de las personas seguidos de sus orígenes, historia, relaciones y publicaciones. Lo que existía eran las enciclopedias impresas que, por lo general, no incluían a personajes tan contemporáneos.

Pero fuera por mi falta de información o por su manera de escribir, yo lo emblanquecí y lo leí como si su conocimiento fuera ahistórico y como si no tuviera ni raza, ni etnia, ya ni se diga todo lo demás de los cruces interseccionales. Pero, ¿por qué leemos así en la Academia? Porque esa es la norma, es lo normal. Y aunque en los tres tomos de Prácticas otras de conocimientos íba/mos en sentido inverso, la obra podría criticarse por la falta de una presencia firme y contundente de autoras negras y/o afrodescendientes y por la ausencia de escritoras posicionadas desde la diversidad sexo-genérica.

Esas dos carencias las fui/mos trabajando, poco a poco, gracias al quehacer dentro de las redes de mujeres que luchan ${ }^{6}$, de feministas, de neozapatistas y de altermundistas. Todo ello de cara a una violencia in crescendo contra las mujeres y las cuerpas diversas, expresada de mil formas, por ejemplo, en los índices de feminicidios en el mundo y las cifras de horror que iban arrojando la homofobia, la lesbofobia y la transfobia. La otra cara de la moneda eran las marchas masivas de jóvenas feministas y de mujeres de pueblos indígenas en las calles de las ciudades de América Latina. Todo eso me/ nos obligó a mirar desde otros ángulos, a sumergirnos en nuestras profundidades de mujeres racializadas rebeldes y a abrazar la disidencia sexual racializada. Al tiempo, en diciembre de 2017, las hermanas zapatistas nos hicieron un fuerte llamado para asistir al Encuentro de Mujeres que Luchan, celebrado el 8 de marzo de 2018 en el Caracol Zapatista de Morelia, y al que asistimos más de 9.000 mujeres de todas partes del mundo.

A unos cuantos meses ya estába/mos en otro territorio autónomo, pero ahora garífuna, en Vallecito, Colón, Honduras. Ahí mujeres y otres garífunas, lenkas y maya xinkas nos enseñaban la importancia de la espiritualidad para la defensa del territorio-cuerpo tierra, para re-tejer la Red de la Vida, como le llaman Lorena Cabnal y la Red de Sanadoras Ancestrales del Feminismo Comunitario Territorial. "Territorio-cuerpo tierra" y "re-tejer la Red de la Vida" no eran simples palabras, ni tampoco las podríamos reducir a conceptos o categorías académicas, eran mucho más que eso, eran prácticas de vida en movimiento para re-existir y sanar en medio de la muerte, el horror y las guerras. Prácticas de vida colectivas enraizadas en la ancestralidad y en la actualidad, que retaban abiertamente el concepto feminista blanco egocentrado de mi cuerpo, mi territorio. Todo eso lo estábamos aprendiendo recorriendo los territorios-cuerpo-tierra indígenas, negros y afros de la mano de ellas y elles. "Para conocer hay que recorrer el territorio", le decían en la década de 1980 los guambianos a los antropólogos solidarios 
que llegaban a sus comunidades a apoyar su emergente movimiento indígena.

Toda esta ebullición de mujeres y diversidades sexo-genéricas estaba teniendo en mí/en nosotras repercusiones onto-epistémico-teórico-políticas que atravesaban desde la cama hasta los centros de investigación. Lo que pensábamos, escribíamos, publicábamos era muy diferente a cuando empezamos a estudiar antropología en un México del desarrollo estabilizador y del boom petrolero. Ahora lo que resonaba en nuestra cabeza era, por ejemplo, que 14 de los 25 países con más feminicidios en el mundo se ubicaban en América Latina y el Caribe. Que, en 2012, El Salvador y Honduras tuvieron las tasas más altas de feminicidios con 14 y 11 asesinadas, respectivamente, por cada 100.000 mujeres (García, 2018). No muy lejano estaba México con 10 mujeres asesinadas por día. En nuestras cabezas retumbaban una y otra vez las consignas: "Ni una más", "Si tocan a una, tocan a todas", "El violador eres tú...."

Fue entonces cuando pusimos juntos escritos insurgidos desde nuestros cuerpos(as) racializados(as) de mujeres de color, indígenas, negras, prietas, mestizas, manchadas, así como lesbo, trans y feministas. Cometimos incluso un sacrilegio pues invitamos a escribir a una feminista blanca, quien reflexionaba desde el privilegio rebelde. Así, en 2020, parimos otro libro colectivo desde nuestra cooperativa autónoma apoyadas en alianzas sororales internacionales. En él dábamos mucho peso a los aportes onto-piestémico-teórico-políticos de las disidencias sexo-genéricas racializadas. Algo poco visibilizado y reconocido incluso dentro de los mismos feminismos.
Al mismo tiempo, en el armado del libro busqué de diferentes maneras agrietar la jerarquía clásica de saber/poder. Al inicio de la obra, en vez de darle un lugar único privilegiado a la feminista argentina decolonial, la tejí para que fuera abrazada por una feminista afrocolombiana y una feminista maya guatemalteca. Las teorías de la feminista no indígena, no afro, sobre las asesinadas de Ciudad Juárez se resignificaron al calor de lo que se nos contaba les sucedía a las mujeres afro en el puerto comercial más grande y violento de Colombia y en una de las regiones indígenas más violentadas en tiempo de la guerra contrainsurgente en Guatemala. Violencia nombrada y juzgada por primera vez en tribunales guatemaltecos como "crímenes de deberes contra la humanidad en la forma de violencia sexual, esclavitud doméstica y sexual".

Estábamos dando un paso muy importante en la Academia y en las ciencias sociales, así como en el activismo y en los feminismos, al poner en el centro las cuerpas rebeldes y en resistencia que, en diferentes geografías y tiempos, con su vida/lucha estaban confrontando de mil formas diversas, simultáneamente al capitalismo, al machismo, al cisheteropatriarcado, al racismo y a las formas dominantes de saber/poder ${ }^{7}$.

\section{Aportes de comunicadoras indígenas en pie de lucha}

El disciplinamiento y la domesticación van de la mano, son hermanos gemelos. Pensemos en nuestros contextos académicos de grado y de posgrado regidos hoy por el capitalismo académico neoliberal. ¿Por qué ahí nuestros procesos de enseñanza-aprendizaje privilegian 
las aulas? ¿Por qué aceptamos sin más que unos "saben" y que otros "no saben"? ¿Por qué en ese espacio solo se puede demostrar que ya "aprendiste algo" a través de textos escritos y no usando otros lenguajes creativos? ¿Por qué los lenguajes audiovisuales son permitidos solo como complemento o ilustración? ¿Por qué tenemos que escribir en lenguas coloniales imperiales cuando $10 \%$ de la población -a nivel nacional en México- y hasta 99\% -en las regiones indígenas- hablan lenguas originarias? ¿Por qué vale mucho publicar en inglés y nada, o casi nada, en las lenguas de los pueblos originarios? La respuesta a cada una de estas preguntas daría para muchos debates, artículos y libros.

Con lo mencionado solo quiero señalar algunas de las formas de comunicación que cotidianamente practicamos en la Academia. En otros contextos las prácticas comunicativas son diametralmente diferentes y con su sola existencia retan y descentran los espacios académicos. Hablemos, por ejemplo, de lo que pasa en la comunicación -en general- y en la comunicación indígena -en particular -. En gramática académica decimos que ambos son conceptos que podrían ser abordados desde dos perspectivas que hoy están vigentes en los pueblos indígenas y afrodiaspóricos en pie de lucha. La primera se basa en la cosmovivencia, la cosmopolítica y la cosmogonía de esos pueblos, es decir, en sus formas de convivir con los diversos seres que buscan la armonía colectiva integral restaurativa. La otra se sustenta en el derecho occidental moderno y se expresa como el "derecho a la comunicación". Unas veces ambas perspectivas coexisten en tensión, conflicto o contradicción; otras se complementan para alcanzar un mismo objetivo. Comunicación y comunicación indígena no tienen una defini- ción única, sino que son términos que están en movimiento y en (re)construcción permanente. Pero sea cual fuere su definición, ello es clave no solo para delinear cómo pensamos sino qué hacemos y cómo lo hacemos.

Ahora bien, para ir a contracorriente de lo establecido como norma en el sistema académico, me parece importante poner en el centro los sentidos y significados de las y los comunicadores indígenas y afrodescendientes de América Latina y no, como suele hacerse, las definiciones de académicos y sus mediaciones sobre esas concepciones.

En la cosmovivencia de los pueblos originarios todo tiene vida $y$, en ese marco, lo que llamamos comunicación adquiere sentidos, modos y formas particulares; por ejemplo, está presente como una experiencia, una vivencia, una práctica sentipensada ancestral, culturalmente situada y de largo aliento. Al respecto, Vilma Almendra, comunicadora del pueblo páez (nasa) de Colombia, integrante de Tejido de Comunicación para la Verdad y la Vida de la Asociación de Cabildos Indígenas del Norte del Cauca (ACIN), nos dice:

La comunicación inicia desde el vientre, desde cuando sentimos las tristezas y alegrías de nuestra madre, desde cuando nos alimentamos con los primeros frutos de nuestra Madre Tierra, desde cuando cruzamos miradas, señas y sueños alrededor del fogón. Nuestra comunicación natural nos identifica como seres colectivos, porque siempre estamos intercambiando, dialogando, sintiendo y expresando en comunidad. (Almendra, 2012, s.p.)

Lo que Vilma Almendra llama "la comunicación propia” tiene que ver con el cómo se es y se está en este mundo, por eso se dice que es ontológica. También es interrelacional pues enfatiza 
la relación no solo entre las personas, sino con todos los seres que habitan la Madre Tierra y el cosmos. Se basa, éticamente hablando, en principios normativos de horizontalidad, reciprocidad y solidaridad. A eso ella también le llama "otra forma de comunicar", que está presente en la vida de los pueblos, pero, agrega:

[...] muchas veces los mismos pueblos indígenas están tan colonizados que cuando hablamos de comunicación hacemos referencia a una cámara, a una grabadora, al internet o al video. Hemos nosotros mismos invisibilizado otra comunicación que es distinta, que nos teje a la vida y a la Madre Tierra, que es esa sabiduría o esa capacidad que debemos desarrollar, revitalizar, para entender, dialogar y escuchar a nuestra Madre Tierra, respetándola. Desde ahí debe partir nuestra otra comunicación. (Almendra, 2013, s.p.)

En esa misma dirección se encuentra la distinción que hace la Organización Nacional Indígena de Colombia, cuando diferencia la comunicación propia de la apropiada. Pero recordemos que Vilma Almendra reflexiona sobre estos temas no en el aire, sino en medio de la celebración, en 2013, de la Cátedra Tata Juan Chávez Alonso, un espacio de convergencia de resistencias anticapitalistas donde las mujeres y los hombres de los pueblos indígenas van más allá de denunciar, se organizan para resistir el embate de la actual ofensiva capitalista patriarcal. Y en ese marco, ella invita a que esa otra comunicación permita a los pueblos entender la "estrategia de ese enemigo", de ese "arriba" que los despoja, saquea, coopta, divide y rompe.

En ese marco Vilma se pregunta: “¿Cómo hacer desde la comunicación dos cosas: arraigar lo cultural histórico a la defensa de la tierra y el territorio [...] [y l]os medios de fuera traerlos para que nos ayuden a defender y acompañar el camino de la palabra libre?" (Almendra, 2013, s.p.) Porque, como afirmó Miriam Miranda, lideresa garífuna de la Organización Fraternal Negra Hondureña, "tenemos que entender que estamos enfrentando una crisis civilizatoria" y que esta crisis "no es solamente una crisis económica, ambiental, política; es una crisis de la civilización occidental moderna". Y -agregóque ese marco nos llama a reflexionar sobre el papel que tiene un comunicador o una comunicadora, negra o indígena, sobre el papel que desempeña "la comunicación formal", refiriéndose a la ejercida por medio, por ejemplo, de la radio o del video frente a la ancestral propia de cada cultura. Luego de compartirnos algunos sucesos acontecidos durante el golpe de Estado en Honduras -realizado en 2009-, concluyó que

[...] hoy el tema de la comunicación es uno de los temas más importantes, más sagrados, porque es el primer poder, ya no es el segundo, ni el tercero, ni el cuarto y puede llegar a definir el rumbo de todo un país, como pasa en Honduras. (Miriam Miranda, Vallecito, Honduras, 1 de septiembre de 2018, comunicación al grupo)

Podría seguir mencionando muchas otras mujeres y hombres comunicadoras de pueblos indígenas y afros para mostrar en dónde están puestos sus énfasis, pero cierro señalando que no estamos frente a "materia prima" para ser analizada por el "experto" académico, sino en diálogo con sujetas epistémicas que no pueden ser reducidas a productoras de meros "testimonios" o "voces". Sus palabras son reflexiones sentipensadas situadas que retan las maneras de ver, hablar y entender características del Poder y la Academia colonial o neocolonial, que siguen teniendo como moneda corriente la ventriloquía: esas formas en las que los no 
indígenas hablan por los indígenas como parte de sistemas de dominación y poder más amplios y complejos ${ }^{8}$.

\section{Insurgencia de saberes zapatistas en tiempos de guerras}

Ahora les voy a contar otro fragmento de este caminar personal-colectivo en busca de la descolonización y la des(hetero)patriarcalización no solo de la Academia y las ciencias sociales, sino de nuestras vidas (así en plural). Es un fragmento de nuestro desaprender de la mano de las, los, loas zapatistas, que nos llevó a cuestionar nuestra noción eurocentrada de guerra. Concepto que aprendemos teóricamente en la universidad y que está cimentado en la forma indoeuropea de nombrar; proviene del alto alemán antiguo werra = desorden, pelea. De esos tiempos a la fecha han pasado siglos, sin embargo, podemos decir que hoy es parte de la episteme dominante concebir la guerra (así en singular) como un combate (armado o no) entre naciones y potencias, pero, ¿qué pasa cuando los pueblos la declaran a un gobierno y a su ejército?, ¿qué pasa cuando las mujeres y los pueblos en resistencia la experimentan en su propio territorio, cuerpo, tierra; cuando, ante ella, crean autonomía de facto y sin permiso? ¿Cómo les impacta a ellxs y a nosotras, así como a nuestros templos del saber/poder?

Como ya mencioné, las zapatistas nos convocaron al Primer Encuentro Internacional, Político, Artístico, Deportivo y Cultural... Lo hicieron de una forma incluyente a través de un nombrarnos como "mujeres que luchan". Ahí entramos todas, sin distingo de raza, diversidad sexo-genérica, edad, clase social, lugar de origen. Ahí estábamos todas: mujeres del color de la Tierra, mujeres blancas y negras, lesbos, bisexuales, trans, queer, darketas, artivistas, cibernautas, campesinas, catequistas, religiosas del abajo, precarias, universitarias, docentes, hijas con sus mamás, lideresas, defensoras de derechos humanos, músicas, teatreras, payasas, bailarinas, esculturistas, fotógrafas, pintoras. Ahí estábamos poniendo nuestra cuerpa para hacer frente al despojo capitalista, machista, heteropatriarcal y racista que experimentamos día a día.

La potencia de ese encuentro tiene muchas aristas, solo anoto una: el caminar del zapatismo se ha dado en medio de una "rotación", un "viraje", no solo del papel y la función del cuerpo femenino o feminizado en las guerras, sino del propio modelo bélico en general, a lo que Rita Segato (2016) llamó las nuevas formas de la guerra. En ellas,

\section{[...] la dominación y la rapiña sexual ya no son, como fueron anteriormente, complementos de la guerra, daños colaterales, sino que [pasan] a ocupar una posi- ción central como arma de guerra productora de cruel- dad y letalidad, dentro de una forma de daño que es simultáneamente material y moral. (p. 59)}

Ese daño funciona como una pedagogía de la crueldad (Segato, 2016) y está expresado en mil formas de violencias hacia las mujeres, las cuerpas diversas, la Madre Tierra y los pueblos. Pero hay algo más que he aprendido caminando y escuchando a las mujeres y otroas zapatistas y a todas las mujeres y cuerpas diversas que luchan. He aprendido a pensar y actuar en el marco de las guerras (así en plural y en femenino). No se trata de un simple giro semántico, por el contrario, implicó moverme del masculinismo abstracto, del logocentrismo, del androcentrismo y abrirme a sentipensar las 
otras guerras en curso: guerras epistemicoteóricopolíticas, guerras energéticoespirituales, guerras intrafamiliares, guerras intrafeminismos, guerras intraizquierdas, guerras cibernéticas, guerras de cifras, guerras de dominación de los hombres (de abajo y de arriba) sobre las mujeres de abajo, como señalan las raperas del colectivo Batallones Femeninos, quienes desde Ciudad Juárez denuncian la violencia contra las mujeres y los feminicidios (Leyva, 2019).

Nada de esto se me hubiera ocurrido si no es por detenerme a aprender la lengua que hablan las compañeras y, en particular, detenerme a escuchar su lengua maya-tseltal. En las lenguas mayenses, y como parte de su realidad onto-epistémica, todo el tiempo se pluraliza y se pone el femenino por delante. Les pongo un ejemplo, al dirigirse en las asambleas a lo que nosotros Ilamaríamos "audiencia", ellos y ellas dicen: ants winiketik ta pisiltik, o sea "mujeres, personas, gente toda". Suele cometerse epistemicidio al traducir winik al español como "hombre" (género masculino), cuando el sentido maya es "persona" (Cumes, 2019) o "gente". Esta suplantación es resultado de la contaminación androcéntrica del español sobre la lengua maya. Ahora conecto esto con el asunto de las guerras. Pluralizar la noción dominante eurocentrada de "la guerra" fue todo un proceso que empezó para mí en 1994. Como verán en seguida, no niego los aportes de los autores académicos, pero al poner en el centro la epistemología, la teoría y la práctica alter y antizapatista, fui/mos más allá de la Academia y sus autores permitidos y legitimados. Veamos un poquito cómo pasó.

El hijo de Castilla la Mancha, Manuel Castells, enfatizó en 1997, en uno de los tres tomos de un libro suyo, que, para difundir su mensaje al mundo, el movimiento zapatista requirió tanto de Internet como de lo que él llamó "una red mundial de grupos de solidaridad". Las nuevas tecnologías de la comunicación jugaban un papel central como infraestructura organizativa del movimiento zapatista, junto con el principio de autenticidad de su propia identidad basada en su especificidad cultural y el deseo de controlar su propio destino. Las ideas de Castells no se quedaron recluidas en la Academia, sino que fueron retomadas por investigadores parte de los llamados think thanks ("tanques de pensamiento") que trabajaban para el Departamento de Defensa de los Estados Unidos. Dichos analistas buscaban entender cómo y por qué un movimiento "local" tenía repercusiones globales y pusieron esos conocimientos al servicio del Pentágono. En ese marco acuñaron el concepto de guerra de redes y, en particular, de guerra de redes sociales zapatistas (Ronfeldt, Arquilla, Fuller \& Fuller, 1998).

Desde un ángulo, podemos decir que el zapatismo estaba contribuyendo a hacer surgir otras formas de entender los conflictos en el nuevo orden global. Desde otro, no podemos dejar de mencionar que los referidos analistas se movían en un campo peligroso que entretejía el servicio de inteligencia, lo militar y lo académico para orientar el actuar del gobierno mexicano y del imperio estadounidense en lo que dieron en llamar "el conflicto en Chiapas". Por su parte, el gobierno mexicano implementó una estrategia de contrainsurgencia en sus facetas de guerra de baja intensidad y de contraguerra a la guerra de redes desde una perspectiva de seguridad nacional subordinada a la doctrina de seguridad nacional estadounidense. Ello puso (y ha puesto) al Ejército Zapatista de Liberación Nacional (EZLN) y a sus solidarixs, hermanadxs y simpatizantes en el centro de la mira del 
ataque social, político, psicológico y militar por parte de las fuerzas gubernamentales, militares y paramilitares.

Los think thanks, los militares, los gobiernos, los políticos, los académicos, todos tenían sus teorías y actuaban conforme a ellas, pero algo a lo que ni el mundo ni la Academia dominante estaban acostumbrados era a que un movimiento campesino indígena político-militar produjera también su propia teoría y que esta se expandiera rápidamente por las venas del mundo inspirando praxis glocal anti y alter. Detengámonos en 1997, el mismo año en que se publicó el tomo que cité de Castells. Entonces, el zapatismo llevó a cabo uno de los análisis políticos más completos y detallados de la situación planetaria; un pensamiento que se adelantaba a su tiempo y a los acontecimientos por venir. Lo que entonces el Subcomandante Insurgente Marcos planteó, hoy, en 2021, es común escucharlo en los espacios de pensamiento crítico radical, pero en 1997 ningún partido o movimiento de izquierda mexicano tenía una lectura similar. Y mucho menos una que fuera acompañada de la creación colectiva, desde abajo, de municipios rebeldes que, desde finales de 1994, empezaron a construir autonomía de facto, retaron al federalismo mexicano, contrarrestaron sociopolíticamente la ocupación militar y paramilitar de Chiapas y dislocaron el principio de máxima ganancia de la economía neoliberal.

El zapatismo afirmó entonces que "la globalización moderna", "el neoliberalismo", debería entenderse, antes que nada, como una nueva guerra de conquista de personas, de territorios, de Estados-nación, a la que llamó IV Guerra Mundial, asumiendo como III Guerra Mundial a la Guerra Fría. A la IV la describió como "la peor y más cruel", pues estaba siendo librada "en todas partes y por todos los medios en contra de la humanidad'. El Subcomandante Insurgente Marcos identificó su modus operandi: por un lado, destrucción/despoblamiento y, por el otro, reconstrucción/reordenamiento. A la vez que advertía que había muchas más, postulaba siete piezas del "rompecabezas neoliberal":

Una [...] la doble acumulación, de riqueza y de pobreza, en los dos polos de la sociedad mundial. La otra [...] la explotación total de la totalidad del mundo. La tercera [...] la pesadilla de una parte errante de la humanidad. La cuarta [...] la nauseabunda relación entre crimen y Poder. La quinta [...] la violencia del Estado. La sexta [...] el misterio de la megapolítica. La [...] la multiforme bolsa de resistencia de la humanidad contra el neoliberalismo.

(Subcomandante Insurgente Marcos, 1997, s.p.)

Ya desde su Primera Declaración de la Selva Lacandona, el EZLN afirmaba que "los dictadores están aplicando una guerra genocida no declarada contra nuestros pueblos desde hace muchos años"9, guerra que remontaba a los tiempos de la conquista y la colonización. Aquí me detengo y señalo que estas formas de nombrar emergieron todas, ciertamente, desde la realidad de lo que, en la década de 1990, se estaba viviendo en los territorios zapatistas: desmantelamientos de los municipios rebeldes, ocupación policíaco-militar, proliferación de grupos paramilitares, la masacre de Acteal, la estigmatización y la expulsión de solidarios extranjeros, la implementación de políticas sociales contrainsurgentes, los ataques al obispo de la diócesis de San Cristóbal y a organismos no gubernamentales locales, etcétera.

Pero si el contexto local explicaba la emergencia de la mirada, fue el crescendo de las violencias, horrores, guerras, muertes, feminicidios, desigualdades en el mundo lo que 
favoreció que, poco a poco, nos tejiéramos, coaligáramos y convergiéramos en lo que hoy recibe el nombre de Travesía por la Vida-Capítulo Europa ${ }^{10}$. Esta es una iniciativa planetaria zapatista y de los pueblos miembros del Congreso Nacional Indígena (CNI) creada en medio de la pandemia como una forma concreta y retadora de seguir la lucha contrahegemónica y el tejido de los mundos otros posibles que ya existen en el planeta Tierra. Es en medio de todo eso que ahora tenemos que trabajar asumiéndonos antes que nada como seres vivos planetarios que, junto a muches otres, luchan por la vida digna justa y plena para todes en medio no solo de la pandemia por COVID-19, sino del colapso civilizatorio en su conjunto.

\section{A manera de conclusión}

Como pudieron apreciar a lo largo de este texto, mi/nuestras prácticas otras de conocimientos han requerido de formas otras de escritura en donde los epicentros cambian, lo tejido es diferente a lo que solemos tejer en un texto académico convencional. La gramática cambia y busca abrirse a una diversidad de lectores que vayan más allá del grupo élite al que pertenecemos. Pero claro, todo esto tiene límites dado que, al final de cuentas, se trata de un texto escrito o digital, en lengua castellana, que hace sentido principalmente a aquellos que habitan la ciudad letrada y las gramáticas ilustradas. Sin embargo, más allá de esa realidad innegable, está la dimensión vivida con la gente en la vida cotidiana y la lucha. Ahí el saber disciplinar coexiste al lado de los saberes femeninos ancestrales y actuales; de los saberes rurales, campesinos, indígenas y populares. Y como hemos consta- tado reiteradas veces, nuestros saberes disciplinares, en ese contexto local, son limitados y no nos permitirían sobrevivir dignamente.

Apenas hace cien años la antropología buscaba entrar en el mapa disciplinar y profesional de las ciencias sociales (Palerm, 2004; Guber, 2014). Ahora, en el segundo decenio del siglo XXI, muchos(as) estudiosos(as) de las ciencias sociales -quienes a la vez están/ mos activos(as) en los movimientos anti, alter, pluri, trans-, con nuestros microhaceres sentipensados creativos-reflexivos, modestamente, están/estamos no solo contribuyendo a agrietar las fronteras disciplinares y a sacar las ciencias sociales del mapa disciplinar, sino que estamos experimentando una y otra vez la mejor vía para la creación de los otros mundos posibles. Como vimos, es en ese devenir que la llamada disciplina antropológica la hemos convertido en una herramienta/instrumento/arma no solo, como decíamos en el siglo XX, para conocer el mundo y transformarlo (así en clave de masculinidad abstracta), sino para construir nosotras mismas (así en clave femenina encarnada) los otros mundos posibles aquí y ahora al faro de los que están ya en construcción: los municipios autónomos zapatistas, las formas alternativas de organización de los pueblos, tribus y naciones que forman el Congreso Nacional Indígena (CNI) y de muchas comunidades, colectivos, redes y movimientos del planeta Tierra. He ahí la pequeña gran diferencia entre el antes y el ahora.

\section{Agradecimientos}

Agradezco a la colega Camila Pascal sus aportes a este texto. 


\section{Notas}

${ }^{1}$ Por siglos, la mitad de la población mundial hemos aceptado que en castellano nos subsuman en el masculino. Para no contribuir a ello usaré, a lo largo del texto, la "x", a veces "(as)" y otras más "e". De esa forma agrietamos el binarismo de género.

${ }^{2}$ Para profundizar ver Leyva \& Köhler (2021) y la obra completa de la que ese capítulo es parte.

${ }^{3}$ Los interesados en profundizar ver Castoriadis Aulagnier (2010 [1975]).

${ }^{4}$ Para conocer los alcances y límites de esta experiencia no sería suficiente leer a Leyva \& Köhler (2021), sino también explorar los aportes de autoridades, jóvenes(as) y comunidades parte de la Pluriversidad Yutsilal Bahlumilal, accesibles en el blog "Educación Agro-eco-visual para florecer autonomía", https://lekilweeliletik. blogspot.com/?zx=ea4c624dfd76795a.

${ }^{5}$ Ellos se pueden descargar libremente en línea: Tomo I (https://www. clacso.org.ar/libreria-latinoamericana/libro_detalle.php?orden=nro_ orden\&id_libro $=1368 \&$ pageNum_rs_libros $=0$ \&totalRows_rs libros=1304\&orden=nro_orden). Tomo II (https://www.clacso.org.ar/

\section{Referencias bibliográficas}

Almendra, v. (2012). Comunicación, cosmovisión y procesos organizativos. Foro Nacional de Comunicación Indígena hacia una Política Pública Diferencial de Comunicación e Información para los Pueblos Indígenas de Colombia, Popayán, 26-30 de noviembre. (2013). Entrevista. Desinformémonos. Cátedra Tata Juan Chávez Alonso, CIDECI Las Casas/Unitierra-Chiapas, Congreso Nacional Indígena, San Cristóbal de Las Casas, Chiapas, 17-18 de agosto.

Castells, M. (1997). The information age: Economy, society, and culture: The power of identity (Vol. II). Oxford: Blackwell Publishers.

Castoriadis Aulagnier, P. (2010 [1975]). La violencia de la interpretación: Del pictograma al enunciado. Buenos Aires: Amorrortu.

Cumes, A. (2019). Colonialismo patriarcal y patriarcado colonial: violencia y despojo en las sociedades que nos dan forma. En Leyva Solano, X. \& Icaza, R. (Coords.), En tiempos de muerte: Cuerpos, rebeldías, resistencias (pp. 297-212). Buenos Aires y San Cristóbal de las Casas: Consejo Latinoamericano de Ciencias Sociales, Cooperativa Editorial Retos, Institut of Social Studies-Erasmus University.

García, A. K. (2018). 14 de los 25 países con más feminicidos se ubican en América Latina. El Economista, 20 de noviembre. Recuperado de https://www.eleconomista.com.mx/politica/14de-los-25-paises-con-mas-feminicidios-se-ubican-en-AmericaLatina--20181120-0048.html

Guber, R. (2014). La generación del conocimiento antropológico: Los trabajos de campo de las antropologías del Sur. III Congreso
libreria-latinoamericana/libro_detalle.php?id_libro=1369\&pageNum_ rs_libros=0\&totalRows_rs_libros $=1304 \&$ orden=nro_orden). Tomo III (https://www.clacso.org.ar/libreria-latinoamericana/ libro_detalle.php?orden=nro_orden\&id_libro=1370\&pageNum_rs_ libros=0\&totalRows_rs_libros $=1304 \&$ orden=nro_orden).

${ }^{6}$ Término acuñado por las mujeres zapatistas.

${ }^{7}$ Se recomienda leer la obra completa (descarga libre en línea: https://www.clacso.org.ar/libreria-latinoamericana/libro_detalle. php?id_libro=1759\&pageNum_rs_libros=1\&totalRows_rs_ libros=1338).

${ }^{8}$ Ver Guerrero (2010) para profundizar en las ventriloquías de los no indígenas sobre los indígenas desde el tiempo colonial y de la hacienda en Ecuador.

${ }^{9}$ Recuperado en Enlace Zapatista, en línea: https://enlacezapatista. ezln.org.mx/1994/01/01/primera-declaracion-de-la-selva-lacandona/.

${ }_{10}$ Para conocer mejor el sentido de la Travesía por la Vida ver en Enlace Zapatista, en línea: https://enlacezapatista.ezln.org. $\mathrm{mx} /$, y la iniciativa colectiva Al Faro Zapatista. Homenaje, en línea: alfarozapatista.jkopkutik.org.

Mexicano de Antropología Social y Etnología, México, 26 de septiembre.

Guerrero, A. (2010). Administración de poblaciones, ventriloquía y transescritura. Quito y Lima: Facultad Latinoamericana de Ciencias Sociales, Instituto de Estudios Peruanos.

Leyva Solano, X. (2019). Abertura. En Leyva Solano, X. \& Icaza, R. (Coords.), En tiempos de muerte: Cuerpos, rebeldías, resistencias (pp. 11-26). Buenos Aires y San Cristóbal de las Casas: Consejo Latinoamericano de Ciencias Sociales, Cooperativa Editorial Retos, Institut of Social Studies-Erasmus University. Recuperado de <https:// www.clacso.org.ar/libreria-latinoamericana/libro_detalle.php?id_ libro=1759\&pageNum_rs_libros=1\&totalRows_rs_libros $=1338 \geq$.

Leyva Solano, X. \& Köhler, A. (2021). Despojos y autonomías de facto en tiempos de pandemia. En Leyva Solano, X., Viera Bravo, P., Trigueiro de Lima, J. M. \& Velázquez Solís, A. C. (Coords.), De despojos y luchas por la vida (pp. 305-331). Buenos Aires y Guadalajara: Consejo Latinoamericano de Ciencias Sociales, Cátedra Jorge Alonso, Universidad de Guadalajara, Cooperativa Editorial Retos. Recuperado de https:/www.clacso.org.ar/libreria-latinoamericana-cm/libro_detalle_ resultado.php?id_libro=2500\&campo=titulo\&texto=De\%20despojos.

Palerm, Á. (2004). Historia de la etnología: Tylor y los profesionales británicos (Vol. 3). México: Universidad Iberoamericana, Instituto Tecnológico y de Estudios Superiores de Occidente.

Pérez, C. (2008). El "crash" de octubre de 2008. El País, 10 de octubre. Recuperado de http://elpais.com/diario/2008/10/11/ economia/1223676001_850215.html. 
Rama, A. 1998. La ciudad letrada. Montevideo: Arca.

Ronfeldt, D., Arquilla, J., Fuller, G. E. \& Fuller, M. (1998). The zapatista social netwar in Mexico. Santa Mónica: RAND Arroyo Center.

Segato, R. L. (2016). La guerra contra las mujeres. Madrid: Traficantes de Sueños.

Subcomandante Insurgente Marcos (1997). 7 piezas sueltas del rompecabezas mundial (El neoliberalismo como rompecabezas: la inútil unidad mundial que fragmenta y destruye naciones). Enlace Zapatista. Recuperado de https://enlacezapatista.ezln.org. mx/1997/06/20/7-piezas-sueltas-del-rompecabezas-mundial-elneoliberalismo-como-rompecabezas-la-inutil-unidad-mundial-quefragmenta-y-destruye-naciones/. 